\title{
The interaction of two inclined cracks with dynamic stress wave loadings
}

\author{
CHWAN-HUEI TSAI ${ }^{1}$ and CHIEN-CHING MA ${ }^{2}$ \\ ${ }^{1}$ Department of Mechanical Engineering, Huafan Institute of Technology, Taipei Hsien, Taiwan 223, Republic of China: \\ ${ }^{2}$ Department of Mechanical Engineering, National Taiwan University, Taipei, Taiwan 107, Republic of China
}

Received 20 February 1991; accepted in revised form 20 March 1992

\begin{abstract}
To gain insight into the phenomenon of the interaction of stress waves with material defects and the linkage of two cracks, the transient response of two semi-infinite inclined cracks subjected to dynamic loading is examined. The solutions are obtained by the linear superposition of fundamental solutions in the Laplace transform domain. The fundamental solution is the exponentially distributed traction on crack faces proposed by Tsai and Ma [1]. The exact closed form solutions of stress intensity factor histories for these two inclined cracks subjected to incident plane waves and diffracted waves are obtained explicitly. These solutions are valid for the time interval from initial loading until the first wave scattered at one crack tip returns to the same crack tip after being diffracted by another crack tip. The result shows that the contribution of diffracted waves to stress intensity factors is much less than the incident waves. The probable crack propagation direction is predicted from the fracture criterion of maximum circumferential tensile stress. The linkage of these two cracks is also investigated in detail.
\end{abstract}

\section{Introduction}

In contrast to static fracture mechanics, both mathematical and experimental difficulties are encountered in the efforts to understand dynamic fracture phenomena. In recent years a considerable amount of research has been directed towards the solution of problems involving wave diffraction by cracks in elastic media in an effort to improve an understanding of the behavior of material failure under dynamic loading, but there still remain some unanswered questions. The initiation, bifurcation and linkage of a main crack and a neighboring micro-crack subjected to dynamic loading is an important phenomenon in material fracture analysis. The interaction of stress waves with cracks is a complicated diffracted process. The diffracted waves emitted from one crack tip will generate mixed mode I and II deformation fields, and cause the other crack to initiate kinking and linking. Numerous experimental investigations using optical techniques [2-5], have been carried out to study parameters such as the stress intensity factor in order to characterize the conditions at the crack tip. According to the experimental observations by Ravi-Chandar and Knauss [2,3], the micro void and flaws will divert the crack to propagate along another plane. In this study, we attempt to simulate the interaction between stress waves and multiple inclined cracks and predict the propagating direction of the cracks.

In conventional studies of a single semi-infinite crack in an unbounded medium subjected to uniformly dynamic loading on the crack faces, the complete solution can be obtained by integral transform methods together with the direct application of the Wiener-Hopf technique [6] and the Cagniard-de Hoop method [7] of the Laplace inversion. If the loading is replaced by a nonuniform distribution having a characteristic length, then the same procedure using integral transformation methods can not be applied directly. Freund [8] studied the problem of an elastic solid containing a half-plane crack subjected to concentrated impact loading on the faces of the crack. To overcome the difficulties of the case with characteristic length, he proposed a 
fundamental solution arising from an edge dislocation climbing along the line ahead of the crack tip with a constant speed. The solution can be constructed by taking an integration over a climbing dislocation of different moving velocities. Basing their procedure on this method, Brock [9-11] and $\mathrm{Ma}$ and Hou $[12,13]$ analyzed a series of problems of a semi-infinite crack subjected to impact loading. Recently, Lee and Freund [14] analyzed fracture initiation of an edge cracked plate subjected to an asymmetric impact.

Because of their complexity, most problems of dynamic fracture that have been solved are restricted to single crack configurations. Jian and Kanwal [15] and Itou [16] investigated the diffraction of two coplanar cracks subjected to normally incident anti-plane shear waves. The dynamic stress field around two noncoplanar cracks subjected to anti-plane shear and inplane waves was solved by Huang and So [17]. Numerical results of scattered fields and elastodynamic stress intensity factors for two neighboring cracks have been obtained from the boundary element method by Zhang and Achenbach [18]. However, all the multiple crack problems mentioned above are restricted to incident periodic stress wave loadings. The problem to be considered in this study is the plane strain transient response of an elastic medium, with two inclined cracks in an arbitrary orientation, subjected to an incident plane longitudinal tensile wave ( $P$ wave) (or transverse shear wave (SV wave)). Particular attention is given to the elastic field near the crack tip, which is completely characterized by the stress intensity factor. The investigation of the idealized semi-infinite cracks can provide some information for an actual elastodynamic fracture problem. It should be noted that while the analysis has been carried out assuming two semi-infinite cracks, the results remain valid for a finite crack up until the time at which waves diffracted from the far tip reach the near tip.

The problem investigated in this study involves multiple characteristic lengths which make a direct solution by standard techniques difficult. Furthermore, none of the methods proposed by Freund [8] and Brock et al. [9] work for this problem. Therefore, some other approach must be followed. A new fundamental solution proposed by Tsai and Ma [1] has been applied successfully in solving multiple characteristic length problems [19-20] and will be used in this study. This alternative fundamental solution is successfully applied to solve the problem and will be demonstrated to be an efficient methodology. The final formulations of dynamic stress intensity factors are expressed explicitly and the effect of each wave is presented in a closed form. The results are valid before the first wave scattered from one crack tip returns to the same crack tip after being diffracted by another crack. Numerical results for elastodynamic stress intensity factors for several configurations have been obtained. Finally, the fracture criterion of maximum circumferential tensile stress proposed by Erdogan and Sih [21] is used to determine the possible direction of crack propagation.

\section{Fundamental solutions}

The mathematical formulation presented in this study is two-dimensional, and for a state of plane strain. As usual in problems of the type considered here, superposition of solutions plays a significant role. The solutions to the problems considered in this study can be determined by superposition of the following problems A and B. Problem A treats the dynamic force acting on a semi-infinite crack; the diffracted waves generated by the crack will induce a traction on 
the planes that will eventually define other crack faces. In problem B, an infinite body containing a semi-infinite crack is considered in which the faces are subjected to tractions which are equal and opposite to those on the corresponding planes in problem A. Unlike usual superposition methods which are performed in the time domain, the superposition scheme proposed in this study is performed in the Laplace transform domain. This methodology allows us to solve more general and difficult problems.

Consider the plane strain deformation of a semi-infinite crack contained in an unbounded medium. An exponentially distributed traction in the Laplace transform domain is applied to the crack faces. The traction force can be divided into a normal force (mode I) and a tangential force (mode II). Because of symmetry with respect to the plane $x_{2}=0$, the problem can be viewed as a half-plane problem with the material occupying the region $x_{2} \geqslant 0$, subject to the boundary conditions

$$
\begin{aligned}
& \bar{\sigma}_{22}\left(x_{1}, 0, p\right)=\mathrm{e}^{p \eta x_{1}}, \quad-\infty<x_{1} \leqslant 0 \\
& \bar{\sigma}_{12}\left(x_{1}, 0, p\right)=0, \quad-\infty<x_{1}<\infty \\
& \bar{u}_{2}\left(x_{1}, 0, p\right)=0, \quad 0 \leqslant x_{1}<\infty
\end{aligned}
$$

for mode I and

$$
\begin{aligned}
& \bar{\sigma}_{22}\left(x_{1}, 0, p\right)=0, \quad-\infty<x_{1}<0, \\
& \bar{\sigma}_{12}\left(x_{1}, 0, p\right)=\mathrm{e}^{p \eta x_{1}}, \quad-\infty<x_{1} \leqslant 0, \\
& \bar{u}_{1}\left(x_{1}, 0, p\right)=0, \quad 0 \leqslant x_{1}<\infty
\end{aligned}
$$

for mode II, where $p$ is the Laplace transform parameter and $\eta$ is a constant. The overbar symbol is used to denote the transform of time $t$. The final results of the dynamic mode $I$ and mode II stress intensity factors in the Laplace transform domain for the boundary conditions (1) and (2) are

$$
\bar{K}_{\mathrm{I}}(p)=-\sqrt{\frac{2}{p}} K_{\mathrm{I}}^{F}(\eta), \quad \bar{K}_{\mathrm{II}}(p)=-\sqrt{\frac{2}{p}} K_{\mathrm{II}}^{F}(\eta)
$$

where

$$
\begin{aligned}
& K_{\mathrm{I}}^{F}(\eta)=\frac{(a+\eta)^{1 / 2}}{(c+\eta) S_{+}(\eta)}, \quad K_{\mathrm{II}}^{F}(\eta)=\frac{(b+\eta)^{1 / 2}}{(c+\eta) S_{+}(\eta)} \\
& S_{ \pm}(\eta)=\exp \left(\frac{-1}{\pi} \int_{a}^{b} \tan ^{-1}\left[\frac{4 \lambda^{2}\left(\lambda^{2}-a^{2}\right)^{1 / 2}\left(b^{2}-\lambda^{2}\right)^{1 / 2}}{\left(b^{2}-2 \lambda^{2}\right)^{2}}\right] \frac{\mathrm{d} \lambda}{\lambda \pm \eta}\right)
\end{aligned}
$$

$a=\sqrt{\rho /(\gamma+2 \mu)}, b=\sqrt{\rho / \mu}, a, b$ and $c$ are the slownesses of the longitudinal wave, shear wave and Rayleigh wave, $\mu$ and $\rho$ the shear modulus and mass density, and $\gamma$ the Lame elastic constant. 


\section{Stress intensity factors of two inclined cracks}

The investigation of defects subjected to oblique incident plane stress waves is an important topic in dynamic fracture analysis. In the time period during which diffracted waves generated by a crack tip have not yet returned to the same crack tip, the problem can be treated as two semi-infinite cracks located in an inclined angle $\bar{\theta}$ as shown in Fig. 1a. The origins of the two coordinate systems $\left(x_{1}, x_{2}\right)$ and $\left(\bar{x}_{1}, \bar{x}_{2}\right)$ are located at the tips of crack $\mathbf{A}$ and crack $\mathbf{B}$, respectively. The crack tip of crack $B$ is located at $x_{1}=l$ and $x_{2}=h$. The coordinate transforms and stress relations between these two systems are

$$
\begin{aligned}
& \bar{x}_{1}=-\left(x_{1}-l\right) \cos \bar{\theta}-\left(x_{2}-h\right) \sin \bar{\theta} \\
& \bar{x}_{2}=\left(x_{1}-l\right) \sin \bar{\theta}-\left(x_{2}-h\right) \cos \bar{\theta} \\
& \sigma_{22}=\sigma_{11} \sin ^{2} \bar{\theta}+\sigma_{22} \cos ^{2} \bar{\theta}-\sigma_{12} \sin 2 \bar{\theta}, \\
& \sigma_{12}=\frac{1}{2}\left(\sigma_{22}-\sigma_{11}\right) \sin 2 \bar{\theta}+\sigma_{12} \cos 2 \bar{\theta} .
\end{aligned}
$$

\subsection{Stress intensity factors of crack $A$ and $B$ subjected to an incident wave}

At time $t=0$, a longitudinal plane tensile wave ( $\mathrm{P}$ wave) or transverse shear wave (SV wave) strikes the crack tip. The position of the plane wavefront prior to time $t=0$ is shown in

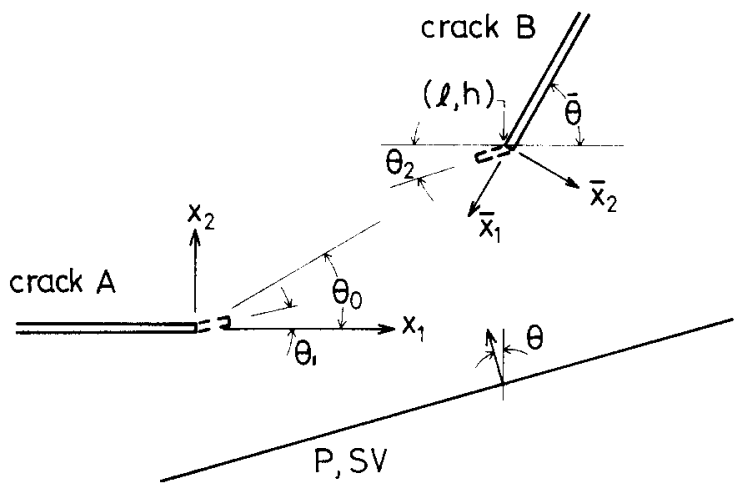

Fig. 1a. Configuration, coordinate system of the two cracks subjected to an incident wave.

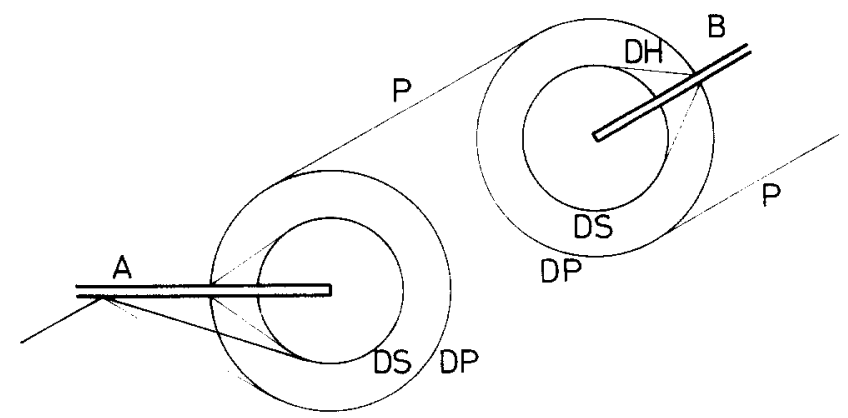

Fig. 1 b. Wave fronts of the two cracks subjected to an incident wave. 
Fig. 1a. $\theta$ is the angle of the normal to the wavefront and the $x_{2}$-axis. The incident $\mathbf{P}$ wave (or SV wave) would give rise to the following stresses

$$
\begin{array}{ll}
\sigma_{\theta \theta}=\sigma_{0} H\left(t+a x_{1} \sin \theta-a x_{2} \cos \theta\right) & \text { for } \mathrm{P} \text { wave, } \\
\sigma_{r \theta}=\sigma_{0} H\left(t+b x_{1} \sin \theta-b x_{2} \cos \theta\right) & \text { for SV wave, }
\end{array}
$$

where $H$ denotes the Heaviside step function. The scattered field of crack A subjected to the incident $\mathrm{P}$ wave (or SV wave) can be obtained by applying the opposite tractions on crack surfaces as follows

$$
\begin{array}{ll}
\sigma_{22}=F_{1} H\left(t+s x_{1}\right), & -\infty<x_{1} \leqslant 0, \\
\sigma_{12}=F_{2} H\left(t+s x_{1}\right) . & -\infty<x_{1} \leqslant 0,
\end{array}
$$

where

$$
\begin{aligned}
& F_{1}=-\sigma_{0}\left(1-\frac{2 a^{2}}{b^{2}} \sin ^{2} \theta\right) \text { for } \mathrm{P} \text { wave } \\
& =-\sigma_{0} \sin 2 \theta \text { for } \mathrm{SV} \text { wave, } \\
& \begin{aligned}
F_{2} & =\sigma_{0} \frac{a^{2}}{b^{2}} \sin 2 \theta \text { for } P \text { wave } \\
& =-\sigma_{0} \cos 2 \theta \text { for } \mathrm{SV} \text { wave, } \\
S & =a \sin \theta \text { for } \mathrm{P} \text { wave } \\
& =b \sin \theta \text { for } \mathrm{SV} \text { wave. }
\end{aligned}
\end{aligned}
$$

The stresses of the scattered field can be obtained by dividing the problem into mode I and mode II deformation fields. The corresponding stress components expressed in the Laplace transform domain are

$$
\begin{aligned}
& \bar{\sigma}_{11}^{\mathrm{I}}\left(x_{1}, x_{2}, p\right)=\frac{1}{2 \pi i p} \int_{B} A_{1}(\lambda) \mathrm{e}^{-p \alpha x_{2}+p \lambda \bar{x}_{1}}+A_{2}(\lambda) \mathrm{e}^{-p \beta x_{2}+p \lambda \bar{x}_{1}} \mathrm{~d} \lambda, \\
& \bar{\sigma}_{12}^{\mathrm{I}}\left(x_{1}, x_{2}, p\right)=\frac{1}{2 \pi i p} \int_{B} A_{3}(\lambda) \mathrm{e}^{-p \alpha x_{2}+p i \bar{x}_{1}}+A_{4}(\lambda) \mathrm{e}^{-p \beta x_{2}+p \lambda \bar{x}_{1}} \mathrm{~d} \lambda, \\
& \bar{\sigma}_{22}^{\mathrm{I}}\left(x_{1}, x_{2}, p\right)=\frac{1}{2 \pi i p} \int_{B} A_{5}(\lambda) \mathrm{e}^{-p \alpha x_{2}+p \lambda \bar{x}_{1}}+A_{6}(\lambda) \mathrm{e}^{-p \beta x_{2}+p \lambda \bar{x}_{1}} \mathrm{~d} \lambda, \\
& \bar{\sigma}_{11}^{\mathrm{II}}\left(x_{1}, x_{2}, p\right)=\frac{1}{2 \pi i p} \int_{B} B_{1}(\lambda) \mathrm{e}^{-p \alpha x_{2}+p \lambda \bar{x}_{1}}+B_{2}(\lambda) \mathrm{e}^{-p \beta x_{2}+p \lambda \bar{x}_{1}} \mathrm{~d} \lambda,
\end{aligned}
$$




$$
\begin{aligned}
& \bar{\sigma}_{12}^{\mathrm{II}}\left(x_{1}, x_{2}, p\right)=\frac{1}{2 \pi i p} \int_{B} B_{3}(\lambda) \mathrm{e}^{-p \alpha x_{2}+p \lambda \bar{x}_{1}}+B_{4}(\lambda) \mathrm{e}^{-p \beta x_{2}+p \lambda \bar{x}_{1}} \mathrm{~d} \lambda \\
& \bar{\sigma}_{22}^{\mathrm{II}}\left(x_{1}, x_{2}, p\right)=\frac{1}{2 \pi i p} \int_{B} B_{5}(\lambda) \mathrm{e}^{-p \alpha x_{2}+p \lambda \bar{x}_{1}}+B_{6}(\lambda) \mathrm{e}^{-p \beta x_{2}+p \lambda \bar{x}_{1}} \mathrm{~d} \lambda,
\end{aligned}
$$

where

$$
\begin{aligned}
& \alpha=\left(a^{2}-\lambda^{2}\right)^{1 / 2}, \quad \beta=\left(b^{2}-\lambda^{2}\right)^{1 / 2}, \\
& A_{1}(\lambda)=\frac{\left(b^{2}-2 \lambda^{2}\right)\left(b^{2}-2 \alpha^{2}\right)}{2 G(\lambda)}, \quad A_{2}(\lambda)=\frac{-2 \lambda^{2} \alpha \beta}{G(\lambda)} \\
& A_{3}(\lambda)=\frac{-\lambda \alpha\left(b^{2}-2 \lambda^{2}\right)}{G(\lambda)}, \quad A_{4}(\lambda)=\frac{\lambda \alpha\left(b^{2}-2 \lambda^{2}\right)}{G(\lambda)} \\
& A_{5}(\lambda)=\frac{\left(b^{2}-2 \lambda^{2}\right)^{2}}{2 G(\lambda)}, \quad A_{6}(\lambda)=\frac{2 \lambda^{2} \alpha \beta}{G(\lambda)} \\
& B_{1}(\lambda)=\frac{-\lambda \beta\left(b^{2}-2 \alpha^{2}\right)}{H(\lambda)}, \quad B_{2}(\lambda)=\frac{-\lambda \beta\left(b^{2}-2 \lambda^{2}\right)}{H(\lambda)} \\
& B_{3}(\lambda)=\frac{2 \lambda^{2} \alpha \beta}{H(\lambda)}, \quad B_{4}(\lambda)=\frac{\left(b^{2}-2 \lambda^{2}\right)^{2}}{2 H(\lambda)}, \\
& H(\lambda)=\frac{F_{2} \sqrt{b+s}}{\left(b^{2}-a^{2}\right)(c+s) S_{+}(s)(b+\lambda)^{1 / 2}(s-\lambda)(c-\lambda) S_{-}(\lambda)} \\
& B_{5}(\lambda)=\frac{-\lambda \beta\left(b^{2}-2 \lambda^{2}\right)}{H(\lambda)}, \quad B_{6}(\lambda)=\frac{\lambda \beta\left(b^{2}-2 \lambda^{2}\right)}{H(\lambda)} \\
& G(\lambda)=\frac{F_{1} \sqrt{a+s}}{\left(b^{2}-a^{2}\right)(c+s) S_{+}(s)(a+\lambda)^{1 / 2}(s-\lambda)(c-\lambda) S_{-}(\lambda)}
\end{aligned}
$$

in which $B$ is the usual inversion path for the two-sided Laplace transform from $\lambda_{1}-i \infty$ to $\lambda_{1}+i \infty$, and $\lambda_{1}$ is a real number located in the interval $\left|\lambda_{1}\right|<a$. The stress intensity factors expressed in the time domain are

$$
K_{\mathrm{I}}(t)=\frac{-2 \sqrt{2} \sqrt{a+s} F_{1}}{\sqrt{\pi}(c+s) S_{+}(s)} \sqrt{t}, \quad K_{\mathrm{II}}(t)=\frac{-2 \sqrt{2} \sqrt{b+s} F_{2}}{\sqrt{\pi}(c+s) S_{+}(s)} \sqrt{t},
$$


for crack $A$, and

$$
\begin{aligned}
& K_{\mathrm{I}}(t)=\frac{-2 \sqrt{2} \sqrt{a+s^{*}} F_{1}^{*}}{\sqrt{\pi}\left(c+s^{*}\right) S_{+}\left(s^{*}\right)} \sqrt{t-t_{0}}, \\
& K_{\mathrm{II}}(t)=\frac{2 \sqrt{2} \sqrt{b+s^{*}} F_{2}^{*}}{\sqrt{\pi}\left(c+s^{*}\right) S_{+}\left(s^{*}\right)} \sqrt{t-t_{0}},
\end{aligned}
$$

for crack B. Where $F_{1}^{*}, F_{2}^{*}$ and $s^{*}$ are obtained by replacing $\theta$ at $F_{1}, F_{2}$ and $s$ by $\bar{\theta}-\theta$. $t_{0}$ in (11) is denoted as $\sqrt{l^{2}+h^{2}} \sin \left(\theta_{0}-\theta\right), \theta_{0}$ is the angle of the line that connect the two crack tips as shown in Fig. 1a.

\subsection{Stress intensity factor of crack $B$ subjected to diffracted waves induced by crack $A$}

Assume that the incident plane $P$ (or SV) wave reaches crack A first, and the diffracted cylindrical waves are generated from the crack tip of crack A. After some time, these diffracted waves will strike crack B. For the traction-free condition in crack surfaces of crack $B$, an additional scattered field must be generated by applying negative traction to the surface of crack B. The stresses in the crack surfaces of crack B induced by the diffracted waves emitted at crack A can be obtained from (8). Taking a coordinate transform from $\left(x_{1}, x_{2}\right)$ to $\left(\bar{x}_{1}, \bar{x}_{2}\right)$ and letting $\bar{x}_{2}=0$, these stresses can be expressed as

$$
\begin{aligned}
& \bar{\sigma}_{22}=\frac{1}{2 \pi i} \int_{B} C_{1}(\lambda) \mathrm{e}^{p(-\lambda \cos \theta+\alpha \sin \theta) \bar{x}_{1}-p \alpha h+p \lambda l}+C_{2}(\lambda) \mathrm{e}^{p(-\lambda \cos \theta+\beta \sin \theta) \bar{x}_{1}-p \beta h+p \hat{\lambda} l} \mathrm{~d} \lambda, \\
& \bar{\sigma}_{\overline{1} \overline{2}}=\frac{1}{2 \pi i} \int_{B} C_{3}(\lambda) \mathrm{e}^{p(-\lambda \cos \bar{\theta}+\alpha \sin \theta) \bar{x}_{1}-p \alpha h+p \lambda l}+C_{4}(\lambda) \mathrm{e}^{p(-\lambda \cos \bar{\theta}+\beta \sin \theta) \bar{x}_{1}-p \beta h+p \lambda l} \mathrm{~d} \lambda,
\end{aligned}
$$

where

$$
\begin{aligned}
& C_{1}(\lambda)=\left(A_{1}+B_{1}\right) \sin ^{2} \bar{\theta}+\left(A_{5}+B_{5}\right) \cos ^{2} \theta-\left(A_{3}+B_{3}\right) \sin 2 \bar{\theta}, \\
& C_{2}(\lambda)=\left(A_{2}+B_{2}\right) \sin ^{2} \bar{\theta}+\left(A_{6}+B_{6}\right) \cos ^{2} \theta-\left(A_{4}+B_{4}\right) \sin 2 \bar{\theta}, \\
& C_{3}=\frac{1}{2}\left(A_{5}+B_{5}-A_{1}-B_{1}\right) \sin 2 \bar{\theta}+\left(A_{3}+B_{3}\right) \cos 2 \bar{\theta} \\
& C_{4}=\frac{1}{2}\left(A_{6}+B_{6}-A_{2}-B_{2}\right) \sin 2 \bar{\theta}+\left(A_{4}+B_{4}\right) \cos 2 \bar{\theta} .
\end{aligned}
$$

It is shown in (12) and (13) that tractions on crack surfaces of crack B that must be negative are represented by the exponential functions $\mathrm{e}^{p(-\lambda \cos \theta+\alpha \sin \theta) x_{1}}$ and $\mathrm{e}^{p(-\lambda \cos \theta+\beta \sin \theta) x_{1}}$. Since the stress intensity factors resulting from applied tractions $e^{p \eta x_{1}}$ are expressed in (3), the solution of this problem can be constructed by the superposition of the fundamental solution obtained in the previous section. Replacing $\eta$ with $-\lambda \cos \bar{\theta}+\alpha \sin \bar{\theta}$ and $-\lambda \cos \bar{\theta}+\beta \sin \bar{\theta}$ and combining (3), (12) and (13), the results for the mixed mode dynamic stress intensity factors in the Laplace 
transform domain for crack $\mathrm{B}$ can be expressed as

$$
\begin{aligned}
\bar{K}_{\mathrm{I}}= & \frac{1}{\sqrt{2} \pi i p^{3 / 2}} \int_{B} C_{1}(\lambda) K_{\mathrm{I}}^{F}(-\lambda \cos \bar{\theta}+\alpha \sin \bar{\theta}) \mathrm{e}^{-p \alpha h+p \lambda l} \\
& +C_{2}(\lambda) K_{\mathrm{I}}^{F}(-\lambda \cos \bar{\theta}+\beta \sin \bar{\theta}) \mathrm{e}^{-p \beta h+p \lambda l} \mathrm{~d} \lambda \\
\bar{K}_{\mathrm{II}}= & \frac{1}{\sqrt{2} \pi i p^{3 / 2}} \int_{B} C_{3}(\lambda) K_{\mathrm{II}}^{F}(-\lambda \cos \bar{\theta}+\alpha \sin \bar{\theta}) \mathrm{e}^{-p \alpha h+p \lambda l} \\
& +C_{4}(\lambda) K_{\mathrm{II}}^{F}(-\lambda \cos \bar{\theta}+\beta \sin \bar{\theta}) \mathrm{e}^{-p \beta h+p \lambda l} \mathrm{~d} \lambda .
\end{aligned}
$$

Finally, the mixed mode stress intensity factors in the time domain can be obtained by employing Cagniard's method for inversing the Laplace transform.

$$
\begin{aligned}
\frac{\pi^{3 / 2}}{2 \sqrt{2}} K_{\mathrm{I}}(t)= & \int_{a r}^{t} \sqrt{t-\tau} \operatorname{Im}\left[C_{1}\left(\lambda_{1}\right) K_{\mathrm{I}}^{F}\left(-\lambda_{1} \cos \bar{\theta}+\alpha \sin \bar{\theta}\right) \frac{\partial \lambda_{1}}{\partial \tau}\right] \mathrm{d} \tau \\
& +\int_{T_{H_{0}}}^{t} \sqrt{t-\tau} \operatorname{Im}\left[C_{2}\left(\lambda_{2}\right) K_{\mathrm{I}}^{F}\left(-\lambda_{2} \cos \bar{\theta}+\beta \sin \bar{\theta}\right) \frac{\partial \lambda_{2}}{\partial \tau}\right] H(b r-\tau) \mathrm{d} \tau \\
& +\int_{b r}^{t} \sqrt{t-\tau} \operatorname{Im}\left[C_{2}\left(\lambda_{3}\right) K_{\mathrm{I}}^{F}\left(-\lambda_{3} \cos \bar{\theta}+\beta \sin \bar{\theta}\right) \frac{\partial \lambda_{3}}{\partial \tau}\right] \mathrm{d} \tau \\
\frac{\pi^{3 / 2}}{2 \sqrt{2}} K_{\mathrm{II}}(t)= & \int_{a r}^{t} \sqrt{t-\tau} \operatorname{Im}\left[C_{3}\left(\lambda_{1}\right) K_{\mathrm{II}}^{F}\left(-\lambda_{1} \cos \bar{\theta}+\alpha \sin \bar{\theta}\right) \frac{\partial \lambda_{1}}{\partial \tau}\right] \mathrm{d} \tau \\
& +\int_{T_{H_{0}}}^{t} \sqrt{t-\tau} \operatorname{Im}\left[C_{4}\left(\lambda_{2}\right) K_{\mathrm{II}}^{F}\left(-\lambda_{2} \cos \bar{\theta}+\beta \sin \bar{\theta}\right) \frac{\partial \lambda_{2}}{\partial \tau}\right] H(b r-\tau) \mathrm{d} \tau \\
& +\int_{b r}^{t} \sqrt{t-\tau} \operatorname{Im}\left[C_{4}\left(\lambda_{3}\right) K_{\mathrm{II}}^{F}\left(-\lambda_{3} \cos \bar{\theta}+\beta \sin \bar{\theta}\right) \frac{\partial \lambda_{3}}{\partial \tau}\right] \mathrm{d} \tau
\end{aligned}
$$

where

$$
\begin{aligned}
& \lambda_{1}=-\frac{\tau}{r} \cos \theta_{0}+i \sqrt{\frac{\tau^{2}}{r^{2}}-a^{2}} \sin \theta_{0}, \quad \lambda_{2}=-\frac{\tau}{r} \cos \theta_{0}-\sqrt{b^{2}-\frac{\tau^{2}}{r^{2}}} \sin \theta_{0}, \\
& \hat{\lambda}_{3}=-\frac{\tau}{r} \cos \theta_{0}+i \sqrt{\frac{\tau^{2}}{r^{2}}-b^{2}} \sin \theta_{0}, \quad T_{H_{0}}=r\left(a\left|\cos \theta_{0}\right|+\sqrt{b^{2}-a^{2}} \sin \theta_{0}\right), \\
& r^{2}=l^{2}+h^{2}, \quad \cos \theta_{0}=\frac{l}{r}, \quad \sin \theta_{0}=\frac{h}{r} .
\end{aligned}
$$


The three terms in (17) and (18) represent the contribution of stress intensity factors from the diffracted longitudinal wave (DP wave), head wave (DH wave), and shear wave (DS wave), respectively. The $\mathrm{DH}$ wave only exists in the region $\cos \theta_{0}<-a / b$. Times equal to $a r, T_{H_{0}}$ and $b r$ represent arrival times of wave fronts fro DP, DH and DS waves, respectively. In the region $-\cos \left(\theta_{0}-\bar{\theta}\right)>a / b$, the branch point $\lambda_{2}=-a \cos \bar{\theta}-\sqrt{b^{2}-a^{2}} \sin \bar{\theta}$ in the second term of (17) and (18) is embraced in the integral path. It represents the reflected head wave from the crack surface generated by the diffracted DS wave, the corresponding arrival time of this wave front is $r\left(-a \cos \left(\theta_{0}-\bar{\theta}\right)-\sqrt{b^{2}-a^{2}} \sin \left(\theta_{0}-\bar{\theta}\right)\right)$.

\subsection{Stress intensity factor of crack $A$ subjected to diffracted waves induced by crack $B$}

Assume the incident $\mathbf{P}$ (or SV) wave will reach crack A first and strike crack $B$ at a later time. Diffracted waves will be generated from crack $\mathbf{B}$ and these cylindrical diffracted waves will propagate to strike crack A. From the same methodology as described above, the stress intensity factors of crack A subjected to a diffracted wave emitted at crack B can be obtained as follows

$$
\begin{aligned}
\frac{\pi^{3 / 2}}{2 \sqrt{2}} K_{\mathrm{I}}(t)= & \int_{a r}^{t-t_{0}} \sqrt{t-t_{0}-\tau} \operatorname{Im}\left[D_{1}\left(\hat{\lambda}_{1}\right) K_{\mathrm{I}}^{F}\left(-\lambda_{1} \cos \bar{\theta}-\alpha \sin \bar{\theta}\right) \frac{\partial \lambda_{1}}{\partial \tau}\right] \mathrm{d} \tau \\
& +\int_{T_{H_{0}}}^{t-t_{0}} \sqrt{t-t_{0}-\tau} \operatorname{Im}\left[D_{2}\left(\lambda_{2}\right) K_{\mathrm{I}}^{F}\left(-\lambda_{2} \cos \bar{\theta}-\beta \sin \bar{\theta}\right) \frac{\partial \lambda_{2}}{\partial \tau}\right] H(b r-\tau) \mathrm{d} \tau \\
& +\int_{b r}^{t-t_{0}} \sqrt{t-t_{0}-\tau} \operatorname{Im}\left[D_{2}\left(\lambda_{3}\right) K_{\mathrm{I}}^{F}\left(-\lambda_{3} \cos \bar{\theta}-\beta \sin \bar{\theta}\right) \frac{\partial \lambda_{3}}{\partial \tau}\right] \mathrm{d} \tau, \\
\frac{\pi^{3 / 2}}{2 \sqrt{2}} K_{\mathrm{II}}(t)= & \int_{a r}^{t-t_{0}} \sqrt{t-t_{0}-\tau} \operatorname{Im}\left[D_{3}\left(\lambda_{1}\right) K_{\mathrm{II}}^{F}\left(-\lambda_{1} \cos \bar{\theta}-\alpha \sin \bar{\theta}\right) \frac{\partial \lambda_{1}}{\partial \tau}\right] \mathrm{d} \tau \\
& +\int_{T_{H_{0}}}^{t-t_{0}} \sqrt{t-t_{0}-\tau} \operatorname{Im}\left[D_{4}\left(\lambda_{2}\right) K_{\mathrm{II}}^{F}\left(-\lambda_{2} \cos \bar{\theta}-\beta \sin \bar{\theta}\right) \frac{\partial \lambda_{2}}{\partial \tau}\right] H(b r-\tau) \mathrm{d} \tau \\
& +\int_{b r}^{t-t_{0}} \sqrt{t-t_{0}-\tau} \operatorname{Im}\left[D_{4}\left(\lambda_{3}\right) K_{\mathrm{II}}^{F}\left(-\lambda_{3} \cos \bar{\theta}-\beta \sin \bar{\theta}\right) \frac{\partial \lambda_{3}}{\partial \tau}\right] \mathrm{d} \tau,
\end{aligned}
$$

where

$$
\begin{aligned}
& D_{1}(\lambda)=\left(A_{1}-B_{1}\right) \sin ^{2} \bar{\theta}+\left(A_{5}-B_{5}\right) \cos ^{2} \bar{\theta}+\left(A_{3}-B_{3}\right) \sin 2 \bar{\theta}, \\
& D_{2}(\lambda)=\left(A_{2}-B_{2}\right) \sin ^{2} \bar{\theta}+\left(A_{6}-B_{6}\right) \cos ^{2} \bar{\theta}+\left(A_{4}-B_{4}\right) \sin 2 \bar{\theta}, \\
& D_{3}=\frac{1}{2}\left(A_{5}+B_{5}-A_{1}-B_{1}\right) \sin 2 \bar{\theta}+\left(A_{3}-B_{3}\right) \cos 2 \bar{\theta}, \\
& D_{4}=\frac{1}{2}\left(A_{6}+B_{6}-A_{2}-B_{2}\right) \sin 2 \bar{\theta}+\left(A_{4}-B_{4}\right) \cos 2 \bar{\theta},
\end{aligned}
$$




$$
\begin{aligned}
& \lambda_{1}=-\frac{\tau}{r} \cos \left(\theta_{0}-\bar{\theta}\right)+i \sqrt{\frac{\tau^{2}}{r^{2}}-a^{2}} \sin \left(\theta_{0}-\bar{\theta}\right), \quad \lambda_{2}=-\frac{\tau}{r} \cos \left(\theta_{0}-\bar{\theta}\right)-\sqrt{b^{2}-\frac{\tau^{2}}{r^{2}}} \sin \left(\theta_{0}-\bar{\theta}\right) \\
& \lambda_{3}=-\frac{\tau}{r} \cos \left(\theta_{0}-\bar{\theta}\right)+i \sqrt{\frac{\tau^{2}}{r^{2}}-b^{2}} \sin \left(\theta_{0}-\bar{\theta}\right) \\
& T_{H_{0}}=-r\left(a\left|\cos \left(\theta_{0}-\bar{\theta}\right)\right|+\sqrt{b^{2}-a^{2}} \sin \left(\theta_{0}-\bar{\theta}\right)\right)
\end{aligned}
$$

\subsection{Probable direction of crack propagation}

In this problem, the deformation field of a crack tip is not only subjected to tension but also to shear loading. In mixed mode experiments, it is usually observed that crack extension takes place at an angle with respect to the original crack. When the mixed stress intensity factors have been obtained, the maximum circumferential tensile stress criterion proposed by Erdogan and Sih [21] is introduced here to examine the crack growth direction. The criterion postulates that the crack will grow in a direction determined by the condition that when the circumferential tensile stress within the asymptotic field is at a maximum, the angle $\theta_{c}$ between the crack line and the direction of crack growth satisfies

$$
\sin \theta_{c} K_{\mathrm{I}}+\left(3 \cos \theta_{c}-1\right) K_{\mathrm{II}}=0
$$

\section{Numerical results}

In the previous sections, the exact dynamic mixed mode stress intensity factor history has been derived. For the numerical calculation of the mixed dynamic stress intensity factors, a Poisson ratio $v$ equal to 0.25 has been chosen. In this case, the ratios of the slownesses are $b=\sqrt{3} a$ and $c=1.88 a$. The first case considered in this study is a horizontal crack A with its crack tip located at $x_{1}=0, x_{2}=0$ and an inclined crack $B$ with its crack tip located at the direction $\theta_{0}=30^{\circ}$ and extended with an inclined angle $\bar{\theta}=30^{\circ}$. An oblique incident $\mathrm{P}$ wave with an oblique angle $\theta=30^{\circ}$ propagates toward the two inclined cracks. At time $t=0$, the incident wave arrives at the crack tips of crack A and B simultaneously, then induces diffracted waves to be generated from the two crack tips. The valid time in this case before the diffracted waves generated from one crack tip return to the same crack tip is $0 \leqslant t / a r \leqslant 2$. The normalized arrival times $t / a h$ of diffracted wave fronts at the crack tip are 1 for the DP wave and $\sqrt{3}$ for the DS wave. The wave fronts of incident and diffracted waves are shown in Fig. $1 \mathrm{~b}$.

The mixed stress intensity factors are given in Fig. 2a which shows that the near tip stress fields along the crack tip line of crack $A$ and $B$ are in a tensile state during the time history valid in this study. Figure $2 \mathrm{~b}$ shows the mode I and mode II stress intensity factor histories for incident SV wave. The mode I stress intensity factor for crack B is very small because the incident SV wave is parallel to crack B. The stress intensity factors are due to the combination of the influence of incident and diffracted waves. From the numerical investigation shown in Fig. 2 for incident $P$ and $S V$ waves, the influence of diffracted waves is estimated to be about 5 percent. Hence the effect of diffracted waves is very small compared with the incident wave. The crack propagation direction predicted by maximum circumferential tensile stress is shown in Fig. 3. 


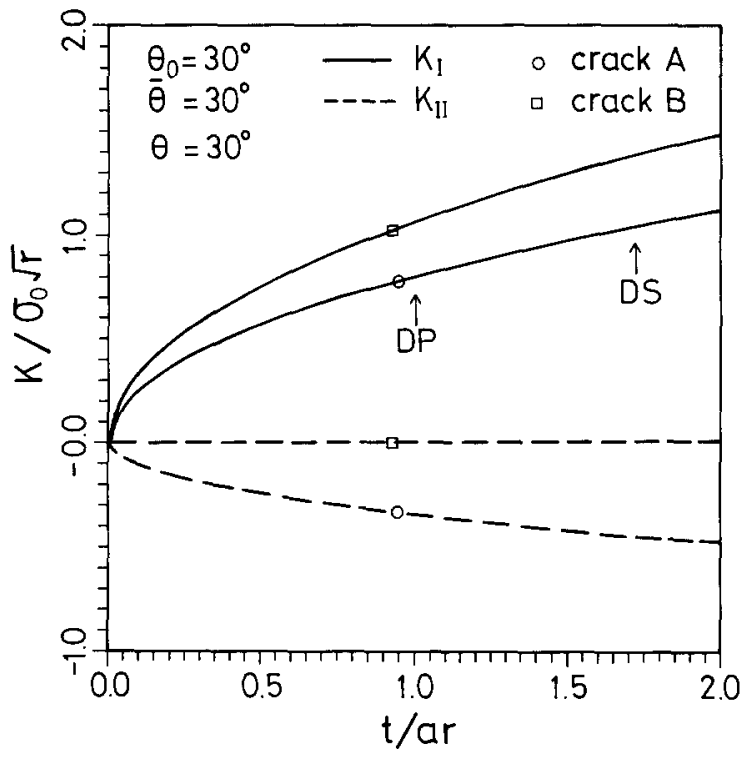

Fig. 2a. Stress intensity factors $K_{\mathrm{I}}$ and $K_{\mathrm{lI}}$ of crack A and $B$ subjected to incident $P$ wave for $\theta_{0}=30^{\circ}$.

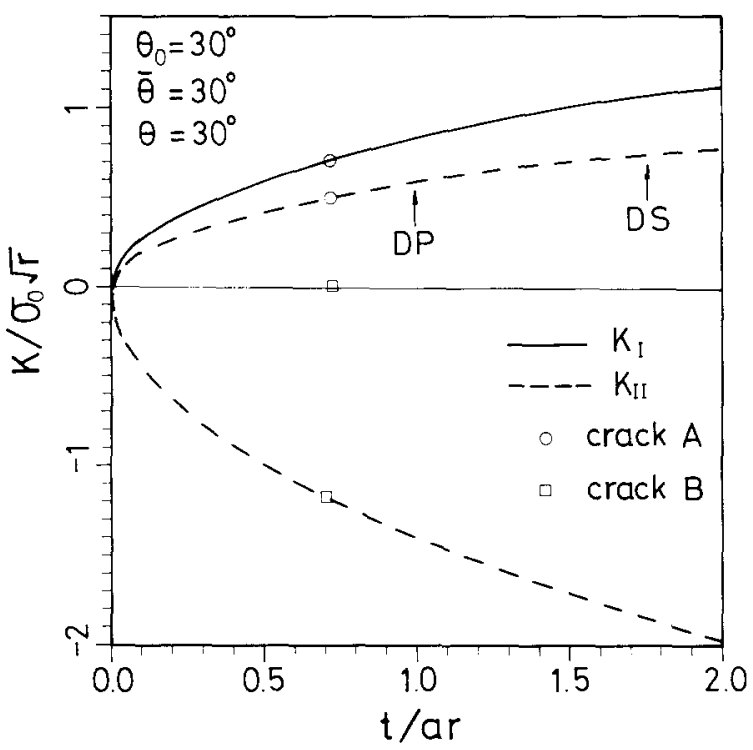

Fig. 2b. Stress intensity factors $K_{1}$ and $K_{11}$ of crack A and $B$ subjected to incident SV wave for $\theta_{0}=30^{\circ}$.

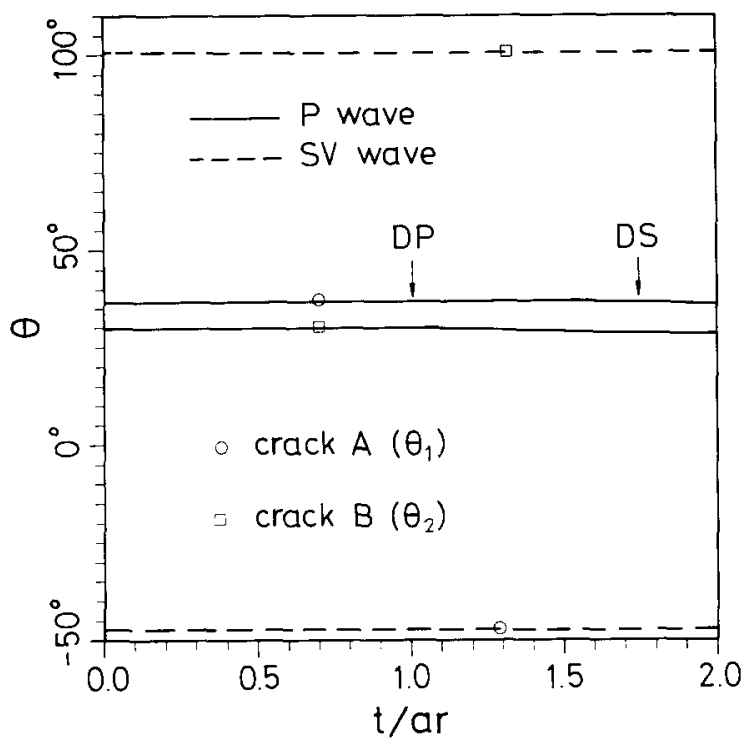

Fig. 3. Prediction of crack propagation direction of crack $\mathrm{A}$ and $\mathrm{B}$ subjected to incident $\mathrm{P}$ and $\mathrm{SV}$ waves for $\theta_{0}=30^{\circ}$.

The values of $\theta_{1}$ and $\theta_{2}$ are denoted as the propagating angles of crack $\mathrm{A}$ and crack $\mathrm{B}$ with respect to the horizontal line. Figure 3 shows that $\theta_{1}$ and $\theta_{2}$ are all positive for incident $P$ wave which means that these two inclined cracks will tend to link together, but the linkage of these two cracks does not occur for incident SV wave.

The second case is a horizontal crack $\mathrm{A}$ and an inclined crack B located at $\theta_{0}=30^{\circ}$; the inclined angle $\bar{\theta}$ is chosen to be $0^{\circ}, 30^{\circ}$ and $60^{\circ}$. In order to understand the influence of the stress intensity factor by diffracted waves, a dynamic impact loading $\sigma_{0}$ is applied uniformly in the surfaces of crack A at time $t=0$. The stress intensity factors of crack B due to the influence of the diffracted waves generated by the tip of crack A are given in Fig. 4. In this loading condition, 


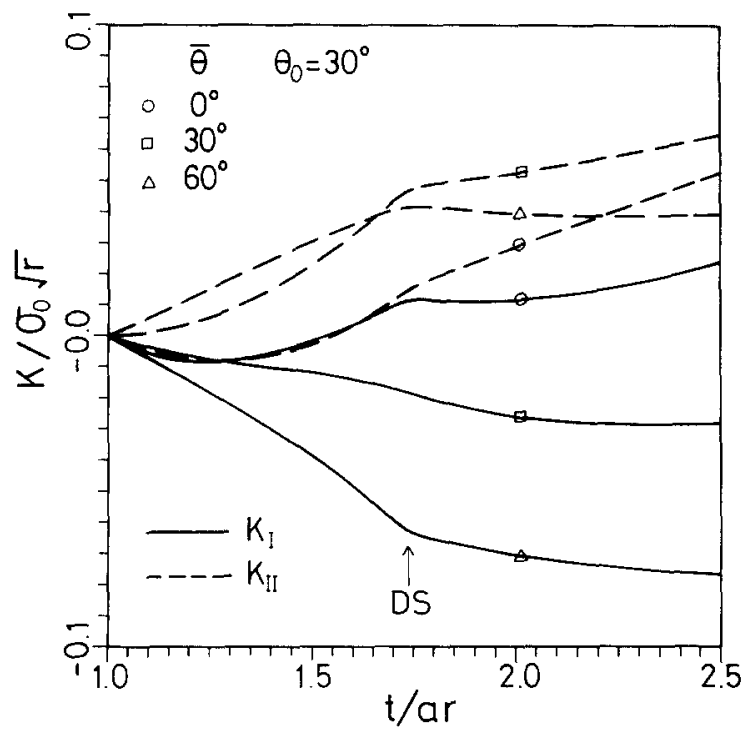

Fig. 4. Stress intensity factors $K_{\mathrm{I}}$ and $K_{\mathrm{II}}$ of crack B subjected to diffracted waves emitted at crack A for $\theta_{0}=30^{\circ}$.

only the effect of diffracted waves emitted at crack A needs to be considered. The value of the stress intensity factor is very small compared to the effect by the incident waves as shown in Fig. 2. The valid time interval in this case is $1<t / a h<3$. The normalized arrival times of diffracted wave fronts at the crack tip of crack B are 1 for the DP wave and $\sqrt{3}$ for the DS wave. The probable crack propagation directions are given in Fig. 5 which shows that crack B will tend to connect with crack $A$ in the case of $\bar{\theta}=0^{\circ}$ and will repulse crack $A$ in the other two cases. It is clear that crack $A$ is dominated by mode $I$ deformation and the influence of diffracted waves emitted from crack B is very small, so that the probable propagation direction of crack A will remain along the $x_{1}$ direction with a negligible variation.

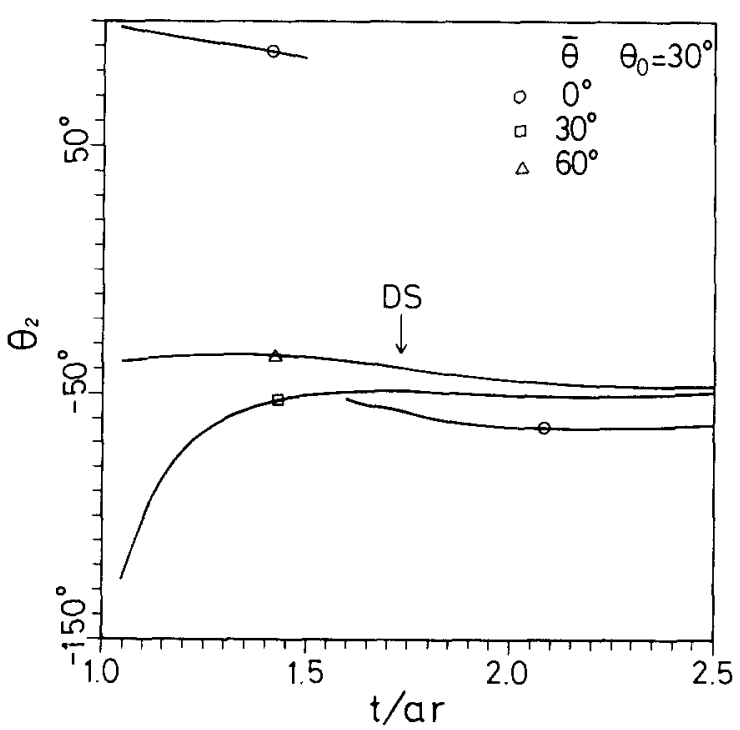

Fig. 5. Prediction of propagation direction of crack B subjected to diffracted wave emitted at crack A for $\theta_{0}=30^{\circ}$. 
The third case considered in this study is the horizontal crack $\mathrm{A}$ and an inclined crack $\mathrm{B}$ located at $\theta_{0}=150^{\circ}$ with the inclined angle $\bar{\theta}$ equal to $180^{\circ}, 150^{\circ}$ and $120^{\circ}$. The geometric configuration and wave fronts of incident wave and diffracted waves is shown in Fig. 6. The incident plane $P$ wave is parallel to the $x_{1}$-axis. Because crack $B$ is located in the shadow zone of crack $A$, the incident wave will not pass through crack $B$ and only diffracted waves emitted from crack A should be considered. The stress intensity factors of crack B subjected to diffracted waves generated by crack $A$ is given in Fig. 7. The stress intensity factors induced from diffracted waves in this case are larger than those in the first and second cases discussed previously. In this case, the diffracted head wave $(\mathrm{DH})$ emitted at the surfaces of crack $A$ will strike the crack tip of crack $\mathrm{B}$. The valid time interval is $1<t / a h<3$, the normalized arrival time of diffracted wave fronts arc 1 for DP wave, 1.573 for DH wavc, and $\sqrt{3}$ for DS wavc. The prediction of the crack propagation direction is shown in Fig. 8. The probable directions are favorable for the linkage to occur.

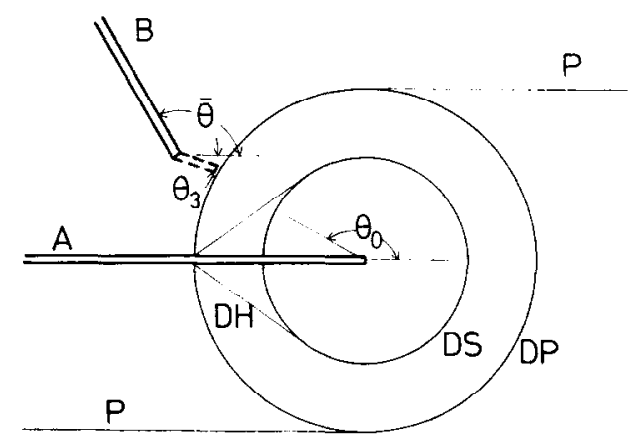

Fig. 6. Wave fronts of the two cracks subjected to an incident wave.

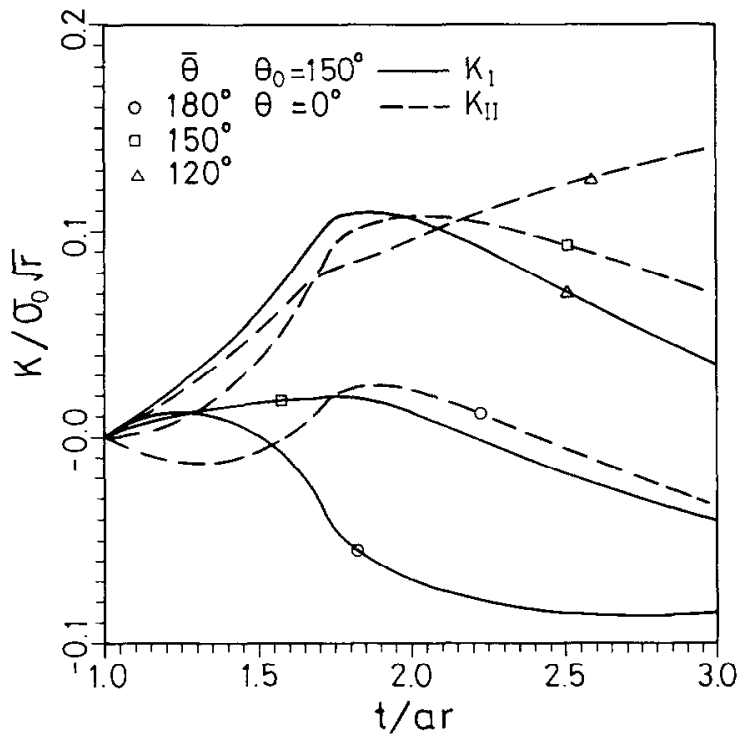

Fig. 7. Stress intensity factors $K_{1}$ and $K_{\mathrm{II}}$ of crack B subjected to diffracted waves emitted at crack A for $\theta_{0}=150^{\circ}$. 


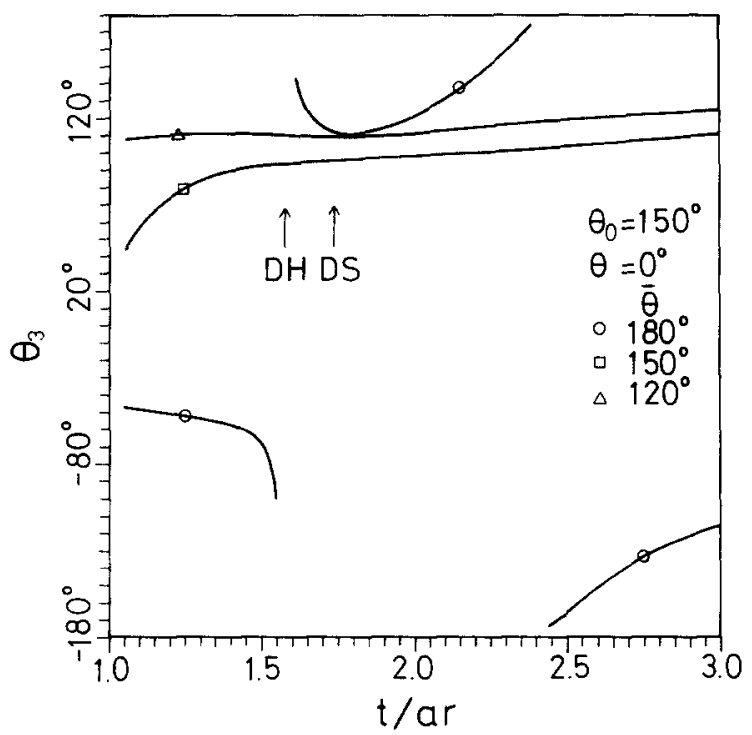

Fig. 8. Prediction of propagation direction of crack B subjected to diffracted wave emitted at crack A for $\theta_{0}=150^{\circ}$.

\section{Conclusion}

The difficulty in determining the transient response in a cracked body subjected to dynamic loading is well known. It will become more complicated and difficult to analyze if two cracks are involved in the analysis. In this study, the transient solution of two inclined cracks subjected to incident plane wave and diffracted waves has been investigated. The dynamic mixed mode stress intensity factors are obtained in an explicit closed form. An exponentially distributed loading on the crack faces in the transform domain is considered as the fundamental solution. Unlike the usual superposition method which is performed in the time domain, the solutions are determined by superposition of the fundamental solution in the Laplace transform domain. This methodology is very powerful in solving more complicated and difficult problems.

The solutions of dynamic stress intensity factors are valid for the time interval from initial loading until the first wave scattered at one crack tip returns to the same crack tip. The dynamic stress intensity factor of one crack due to the influence of the incident plane wave and the diffracted waves generated from another crack tip has been examined in detail. From the numerical results obtained in this study, it can be concluded that the stress intensity factors due to the diffracted waves emitted at another crack are less influential than the incident wave.

Leaving aside the possibility of the compression state being generated in the near tip, the crack propagation direction is predicted based on the criterion of maximum circumferential tensile stress. The prediction shows that these two cracks would mostly tend to approach each other, which means the linkage of these two cracks will occur. However, the investigation of this study has shown that influence of an incident plane wave on stress intensity factors is much larger than diffracted waves emanated from crack tips. Hence the direction of a crack extension is highly dependent on the angle between a crack face and propagation direction of the incident wave. The transient response of a stationary crack subjected to diffracted waves generated by a propagating crack also has been investigated and will be discussed in a follow-up report. 


\section{Acknowledgment}

The work described here was supported by the National Science Council (Republic of China), through grant NSC 79-0401-E-002-36 to National Taiwan University. This research support is gratefully acknowledged.

\section{References}

1. C.H. Tsai and C.C. Ma, Journal of Applied Mechanics (1992) in press.

2. K. Ravi-Chandar and W.G. Knauss, International Journal of Fracture 26 (1984) 65-80.

3. Ibid, 141-154.

4. A.J. Rosakis, J. Duffy and L.B. Freund, Journal of the Mechanics and Physics of Solids 32 (1984) 443-460.

5. R.V. Mahajan and K. Ravi-Chandar, International Journal of Fracture 41 (1989) 235-252.

6. B. Noble, The Wiener-Hopf Technique, Pergamon Press (1958).

7. A.T. de Hoop, 'Representation Theorems for the Displacement in an Elastic Solid and Their Application to Elastodynamic Diffraction Theory', Doctoral dissertation, Technische Hogeschool, Delft (1958).

8. L.B. Freund, International Journal of Engineering Science 12 (1974) 179-189.

9. L.M. Brock, International Journal of Solids and Structures 18 (1982) 467-477.

10. L.M. Brock, Journal of Elasticity 14 (1984) 415-424.

11. L.M. Brock, M. Jolles and M. Schroedl, Journal of Applied Mechanics 52 (1985) 287-290.

12. C.C. Ma and Y.C. Hou, Journal of Engineering Science 28 (1990) 1321-1329.

13. C.C. Ma and Y.C. Hou, Journal of Applied Mechanics 58 (1991) 703-709.

14. Y.J. Lee and L.B. Freund, Journal of Applied Mechanics 57 (1990) 104-111.

15. D.L. Jain and R.P. Kanwal, International Journal of Solids and Structures 8 (1972) 961-975.

16. S. Itou, International Journal of Solids and Structures 16 (1980) 1147-1153.

17 J.Y. Huang and H. So, Engineering Fracture Mechanics 29 (1988) 335-347.

18. C.H, Zhang and J.D. Achenbach, Journal of Applied Mechanics 55 (1988) 104-110.

19. C.H. Tsai and C.C. Ma, International Journal of Solids and Structures (1992) in press.

20. C.H. Tsai and C.C. Ma, International Journal of Solids and Structures (1992), submitted.

21. F. Erdogan and G.C. Sih, Journal of Basic Engineering 85 (1963) 519-527. 